〔Med. Entomol. Zool. Vol. 52 No. 3 p. 231-239 2001]

\title{
A method for estimating food preference of the commensal rat
}

\author{
Yasutada ITO ${ }^{1), 2)}$ \\ 1) Department of Environmental Biology, Japan Environmental Sanitation Center, \\ Yotsuyakamicho 10-6, Kawasaki-ku, Kawasaki, 210-0828 Japan \\ 2) Department of Tropical Medicine and Parasitology, Dokkyo University School of Medicine, \\ Mibu, Tochigi, 321-0293 Japan
}

(Received: 28 September 2000; Accepted: 17 August 2001)

Key words: food preference, acceptability, unpoisoned bait, index, Rattus rattus, Rattus norvegicus

\begin{abstract}
A new index concerning acceptability of unpoisoned baits was worked out and applied to study the food preference of the roof rat, Rattus rattus (Linnaeus, 1758) and the Norway rat, Rattus norvegicus (Berkenhout, 1769). The index is shown as a formula, Acceptability (A)-index $=\{(x-y) /(x+y)\} \times 100$, where $x$ and $y$ are the average intakes of alternative baits. Then, the formula was tested for the preferences in placement, coloration and kinds of baits. Both of these species showed the tendency to take the bait in one side more than the other side, and the average of absolute values of the A-index was as high as 22.1 in the roof rat and 17.9 in the Norway rat, respectively. Therefore, in the other choice tests, I exchanged the position of two baits daily to minimize partiality due to the influence of the food position. In the choice test on coloration of baits, there was little difference between red-colored and uncolored seeds in the intake of the roof rat (average A-index $=-5.4$ ). Although the Norway rat had a tendency to prefer red to uncolored seed (average A-index = 17.8), the sample size of the A-index was only 5 , and the $95 \%$ confidence limit was considerably wide. Therefore, it seemed that there was minimal effect of red coloration on the rats. In the choice test between whole rice flour and mixed powdered feed, although the roof rats showed the tendency to take one of two feed types, they entirely preferred the latter to the former (average A-index $=-21.9$ ). In conclusion, the A-index could be used as a method for estimating food preference of the commensal rat, and, moreover, it is hoped that the A-index would be used to estimate the acceptability of rodenticides in choice tests of the rats.
\end{abstract}

\section{INTRODUCTION}

The commensal rats, Rattus spp., especially the roof rats, Rattus rattus (Linnaeus, 1758) seldom eat poisoned baits. When the ten roof rats were given a poisoned bait containing $0.025 \%$ warfarin and an unpoisoned bait for ten days, they ate less of the former than the latter, and did not die at all (Ito, 1998). Therefore, it is necessary to improve their acceptability of poisoned baits for successful control. We should carry out many patterns of choice tests with several rodenticides and bait materials. For this purpose, it is hoped to make up an index to estimate the food preference of the commensal rats.

There are several reports on food preference in choice tests of the Norway rats, Rattus norvegicus (Berkenhout, 1769) in Japan (Ogushi and Tokumitsu, 1970; Naganuma et al., 1973; Ikeda et al., 1976), and of the roof rats (Prakash et al., 1980; Ito et al., 1982). However, all of these reports used only average or percent bait consumption to estimate food preference, whereas Nahas et al. (1989) have used the 
index, palatability $(\%)=\{\mathrm{P} /(\mathrm{P}+\mathrm{C})\} \times 100$ (P:consumption of poisoned baits, C: consumption of control baits). I worked out a new index (A-index) to estimate food preference of unpoisoned baits, and the Aindex would be used for comparing acceptability of several rodenticides in the choice tests of the commensal rats.

\section{Materials And Methods}

\section{Test animals}

The roof rats were captured with sticky traps at large buildings in Tokyo and Yokohama, and were used in this study after they were bred in the laboratory for about one month until their health was recovered. The Norway rats were live-trapped at a landfill site in Tokyo, and they and their progeny were used. Ten or five rats of each species were weighed, sexed and isolated in a metal test cage measuring 39 $\times 26 \times 20(\mathrm{H}) \mathrm{cm}$ for at least one day before the tests.

\section{Test conditions}

The rats were provided with a laboratory diet (Oriental Yeast Co., MF) and water ad libitum. The room temperature was kept at $23 \pm 2{ }^{\circ} \mathrm{C}$. Mixed powdered feed (Oriental Yeast Co., MF), sunflower seeds (made in China) and whole rice flour were materials for baits.

\section{Test methods}

I carried out three choice tests in an apparatus shown in Fig. 1. The roof rat or the Norway rat were daily given $20 \mathrm{~g}$ and $30 \mathrm{~g}$ of bait in each of two food containers, respectively. The amount of bait eaten from each container was measured daily.

1. Placement of baits (Test 1).

Mixed powdered feed was given in two containers, $10 \mathrm{~cm}$ in diameter and $5 \mathrm{~cm}$ in height, with a $4.5 \mathrm{~cm}$ circular opening in the center (Fig. 1).

\section{Coloration (Test 2).}

In the test of roof rats, a $200 \mathrm{ml}$ water
Test cage

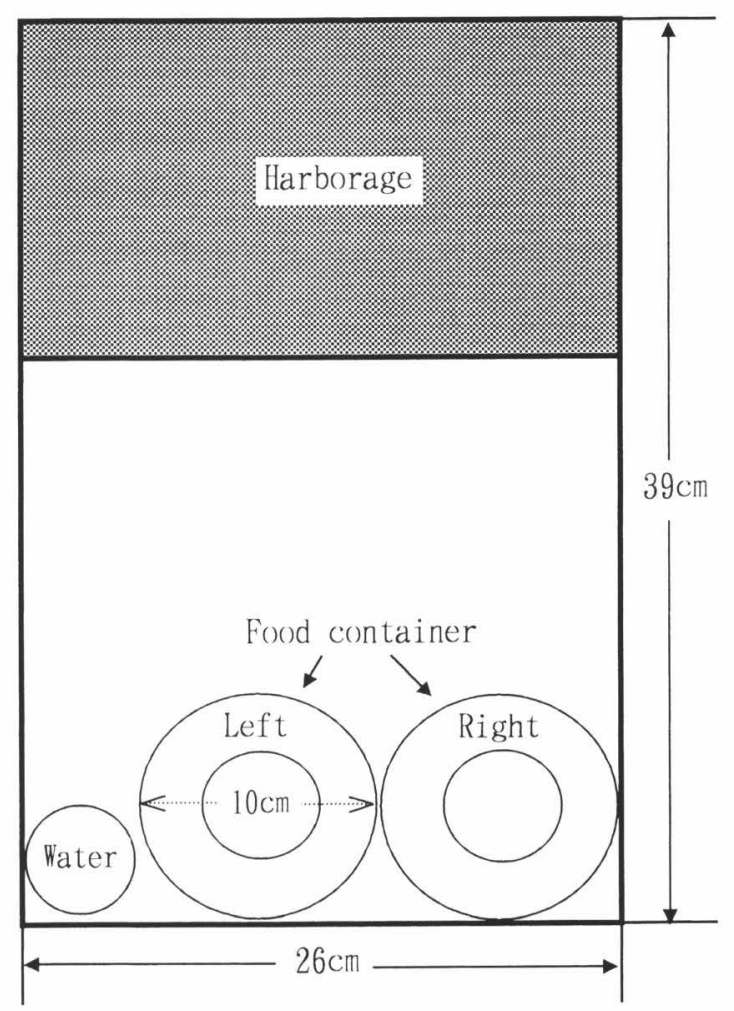

Fig. 1. Test apparatus. The roof or Norway rat were daily given $20 \mathrm{~g}$ and $30 \mathrm{~g}$ of bait in two food containers, respectively.

solution $(0.2 \%)$ of a food additive (red No. 2) was mixed in $1.6 \mathrm{~kg}$ of sunflower seeds. Similarly in Norway rats, a $400 \mathrm{ml}$ water solution $(0.4 \%)$ of red No. 2 was mixed in $1.6 \mathrm{~kg}$ of mixed powdered feed. Then, the red-colored baits and uncolored baits were given in the same manner as Test 1.

\section{Kinds of baits (Test 3).}

Mixed powdered feed and whole rice flour were provided in the same containers as Test 1. The amount of bait eaten from each container was measured daily, the positions of the baits being reversed after one day to reduce the possible effects of place preference in Tests 2 and 3.

\section{Estimate methods}

In the three choice tests, each A-index was calculated with the following formu- 
la.

A-index $=\{(x-y) /(x+y)\} \times 100$, where $x$ and $y$ were average intake in one and the other side, respectively.

The A-index changes from -100 to 100 when $x=0$ or $y=0$ respectively. When the total of two average intakes was less than $1 \mathrm{~g}$, the data was not adopted. In Tests 1 , 2 and $3, \mathrm{~L}$ and $\mathrm{R}$ or $\mathrm{R}$ and $\mathrm{U}$ or $\mathrm{W}$ and $\mathrm{M}$ were used instead of $x$ and $y$. L and $\mathrm{R}$ indicated average intake in the left or right side, $\mathrm{R}$ and $\mathrm{U}$ indicated average intake of red or uncolored bait, and $\mathrm{W}$ and $\mathrm{M}$ indicated average intake of whole rice flour or mixed powdered feed, respective$1 y$.

\section{REsults AND Discussion}

\section{Test 1}

The roof rat and the Norway rat showed a tendency to prefer the bait in one side. The average of absolute values of the Aindex was 22.1 and 17.9 , respectively (Tables 1 and 2). As a result of Test 1, it was found that the two species of rats had a tendency to show a difference in position of placement as to the intake of bait, even if two baits were the same.

Jain and Pawde (1981) have reported that as place preference effect, the differences in consumption recorded from the 2 baiting points at each station, were significant in the field test against the roof rat. Shafi et al. (1992) have described that the position of feeding cups was changed daily to avoid any place preference trend. We should exchange the position of two baits daily in the choice tests.

\section{Test 2}

In the case of the roof rat, since the rat (No. 1) ate a very small amount such as only $0.4 \mathrm{~g}$ in total of two average intakes, the data was not adopted. The average of A-indexes was as low as -5.4 (Table 3 ). In the case of the Norway rat, the average of A-indexes was as high as 17.8 (Table 4). However, sample size of the A-index was only 5 , and $95 \%$ confidence limit was con- siderably wide, containing zero (Table 6). The rat (No. 5) significantly preferred red feed to uncolored, showing A-index of 58.5. Therefore, in this test, it was not possible mention that the Norway rat preferred red feed.

\section{Test 3}

The roof rat had a tendency to prefer either mixed powdered feed or whole rice flour. Six rats preferred mixed powdered feed and three rats preferred whole rice flour. Finally, the average of A-indexes was as low as -21.9 , because more rats preferred mixed powdered feed to whole rice flour (Table 5). Further in Test 2 and Test 3, A-indexes concerning the left and right sides were calculated to examine the difference in both sides. As a result, the averages of A-indexes were 1.2, - 26.5, 2.1 about Tables 3, 4 and 5, respectively. There was little difference in placement of baits in Tables 3 and 5, and especially the kinds of bait influenced on the intakes of rats more than the placement of bait in Table 5. It was thought that the result of Table 4 was exceptional.

In this study, I examined food preference of unpoisoned baits using a new index (A-index). The figures concerning A-index are summarized in Table 6 , that is to say sample size, average, $\mathrm{SD}, \mathrm{SE}$, and 95\% confidence limit. Palatability index (Nahas et al., 1989) takes values from 0 to 100 , comparing with the A-index which takes a wider range of values from -100 to 100 . The A-index would be used to compare the data on food preference of rats, and, moreover, the A-index might be used for estimating acceptability of poisoned baits containing rodenticides in the same manner as the Palatability index.

\section{ACKNOWLEDGMENTS}

I wish to thank Dr. Ikuo Tanaka, Japan Environmental Sanitation Center, for reading the manuscript at first. I am also very grateful to Prof. Hajime Matsuda, Dokkyo University School of Medicine, and Prof. 
Table 1. Daily intake and A-index of two same mixed powdered feed in the choice test in Rattus rattus.

\begin{tabular}{|c|c|c|c|c|c|c|c|c|c|c|c|c|c|c|c|c|c|c|c|c|c|}
\hline \multirow{3}{*}{$\begin{array}{l}\text { No. } \\
\text { of } \\
\text { rats }\end{array}$} & \multirow{3}{*}{$\begin{array}{l}\text { Body } \\
\text { weight } \\
\text { (g) }\end{array}$} & \multirow{3}{*}{ Sex } & \multicolumn{16}{|c|}{ Daily intake (g) } & \multirow{2}{*}{\multicolumn{2}{|c|}{$\begin{array}{l}\text { Average } \\
\text { intake } \\
\text { (g/day) }\end{array}$}} & \multirow{3}{*}{ A-index } \\
\hline & & & \multicolumn{2}{|c|}{1} & \multicolumn{2}{|c|}{2} & \multicolumn{2}{|c|}{3} & \multicolumn{2}{|c|}{4} & \multicolumn{2}{|c|}{5} & \multicolumn{2}{|c|}{6} & \multicolumn{2}{|c|}{7} & \multicolumn{2}{|c|}{8} & & & \\
\hline & & & $\mathrm{L}$ & $\mathrm{R}$ & $\mathrm{L}$ & $\mathrm{R}$ & $\mathrm{L}$ & $\mathrm{R}$ & $\mathrm{L}$ & $\mathrm{R}$ & L & $\mathrm{R}$ & $\mathrm{L}$ & $\mathrm{R}$ & $\mathrm{L}$ & $\mathrm{R}$ & $\mathrm{L}$ & $\mathrm{R}$ & $\mathrm{L}$ & $\mathrm{R}$ & \\
\hline 1 & 153 & $\sigma^{7}$ & 1.1 & 0.4 & 3.0 & 1.2 & 4.7 & 3.9 & 4.7 & 3.5 & 5.5 & 4.6 & 6.4 & 3.8 & 4.1 & 3.0 & 6.1 & 1.4 & 4.5 & 2.7 & 25.0 \\
\hline 2 & 173 & $\sigma^{7}$ & 1.1 & 2.8 & 3.3 & 3.5 & 2.5 & 3.6 & 2.2 & 3.6 & 2.6 & 5.0 & 2.0 & 5.8 & 0.5 & 5.5 & 1.2 & 5.1 & 1.9 & 4.4 & 39.7 \\
\hline 3 & 173 & $\sigma^{7}$ & 4.3 & 2.2 & 5.7 & 2.8 & 5.4 & 4.7 & 4.0 & 7.2 & 4.8 & 4.7 & 7.7 & 3.0 & 4.5 & 4.1 & 4.6 & 3.8 & 5.1 & 4.1 & 10.9 \\
\hline 4 & 200 & $\sigma^{\pi}$ & 0.0 & 0.0 & 1.0 & 0.7 & 2.3 & 0.4 & 5.7 & 0.0 & 6.3 & 0.9 & 4.0 & 6.0 & 5.8 & 1.9 & 7.4 & 1.5 & 4.1 & 1.4 & 49.1 \\
\hline 5 & 210 & $\sigma^{\pi}$ & 3.7 & 5.6 & 3.2 & 5.7 & 4.8 & 3.4 & 2.6 & 3.9 & 2.4 & 5.6 & 4.7 & 5.7 & 6.8 & 1.4 & 4.4 & 3.6 & 4.1 & 4.4 & 3.5 \\
\hline 6 & 90 & 우 & 4.4 & 3.8 & 4.1 & 2.6 & 5.1 & 3.4 & 4.6 & 4.0 & 4.8 & 4.9 & 5.3 & 3.7 & 4.5 & 3.5 & 4.6 & 3.7 & 4.7 & 3.7 & 11.9 \\
\hline 7 & 99 & 우 & 1.6 & 6.6 & 3.2 & 6.1 & 3.0 & 6.3 & 4.7 & 5.2 & 3.4 & 5.2 & 4.2 & 4.2 & 2.9 & 3.9 & 0.4 & 7.3 & 2.9 & 5.6 & 31.8 \\
\hline 8 & 127 & 우 & 3.6 & 2.5 & 5.9 & 1.9 & 5.4 & 2.4 & 5.6 & 1.8 & 4.2 & 3.4 & 6.2 & 1.2 & 4.6 & 1.8 & 2.5 & 6.4 & 4.8 & 2.7 & 28.0 \\
\hline 9 & 132 & 우 & 0.0 & 0.0 & 0.8 & 0.4 & 2.3 & 3.0 & 3.0 & 4.4 & 3.6 & 4.2 & 4.8 & 3.3 & 2.2 & 3.6 & 2.5 & 4.0 & 2.4 & 2.9 & 9.4 \\
\hline 10 & 182 & 우 & 0.6 & 0.6 & 2.1 & 2.6 & 3.0 & 2.5 & 4.5 & 3.7 & 6.8 & 3.3 & 6.3 & 5.5 & 4.8 & 3.8 & 3.1 & 3.0 & 3.9 & 3.1 & 11.4 \\
\hline
\end{tabular}

L, left side; $\mathrm{R}$, right side.

Average

22.1

A-index of average intake $=\{(L-R) /(L+R)\} \times 100$.

A-indexes were shown by absolute value because same mixed powdered feed was used in two containers. 
Table 2. Daily intake and A-index of two same mixed powdered feed in the choice test in Rattus norvegicus.

\begin{tabular}{|c|c|c|c|c|c|c|c|c|c|c|c|c|c|c|c|c|c|c|c|c|c|}
\hline \multirow{3}{*}{$\begin{array}{l}\text { No. } \\
\text { of } \\
\text { rats }\end{array}$} & \multirow{3}{*}{$\begin{array}{l}\text { Body } \\
\text { weight } \\
\text { (g) }\end{array}$} & \multirow{3}{*}{ Sex } & \multicolumn{16}{|c|}{ Daily intake (g) } & \multirow{2}{*}{\multicolumn{2}{|c|}{$\begin{array}{l}\text { Average } \\
\text { intake } \\
\text { (g/day) }\end{array}$}} & \multirow{3}{*}{ A-index } \\
\hline & & & \multicolumn{2}{|c|}{1} & \multicolumn{2}{|c|}{2} & \multicolumn{2}{|c|}{3} & \multicolumn{2}{|c|}{4} & \multicolumn{2}{|c|}{5} & \multicolumn{2}{|c|}{6} & \multicolumn{2}{|c|}{7} & \multicolumn{2}{|c|}{8} & & & \\
\hline & & & $\mathrm{L}$ & $\mathrm{R}$ & $\mathrm{L}$ & $\mathrm{R}$ & L & $\mathrm{R}$ & $\mathrm{L}$ & $\mathrm{R}$ & $\mathrm{L}$ & $\mathrm{R}$ & L & $\mathrm{R}$ & $\mathrm{L}$ & $\mathrm{R}$ & L & $\mathrm{R}$ & $\mathrm{L}$ & $\mathrm{R}$ & \\
\hline 1 & 271 & $\sigma^{7}$ & 7.7 & 2.3 & 8.6 & 4.8 & 10.5 & 4.4 & 10.7 & 5.9 & 10.6 & 2.7 & 10.4 & 2.3 & 7.8 & 3.8 & 10.6 & 3.1 & 9.6 & 3.7 & 44.4 \\
\hline 2 & 279 & $0^{x}$ & $\mathrm{~N}$ & $\mathrm{~N}$ & 5.9 & 11.5 & 2.7 & 10.0 & 1.4 & 16.6 & 0.5 & 18.8 & 2.3 & 17.2 & $\mathrm{~N}$ & $\mathrm{~N}$ & 21.5 & 12.6 & 5.7 & 14.5 & 43.6 \\
\hline 3 & 321 & $0^{7}$ & 1.8 & 2.2 & 8.0 & 3.5 & 10.0 & 2.6 & 9.8 & 6.0 & 6.4 & 8.0 & 8.4 & 6.6 & 8.6 & 2.9 & 9.9 & 4.0 & 7.9 & 4.5 & 27.4 \\
\hline 4 & 364 & $0^{7}$ & 7.2 & 3.0 & 11.2 & 6.0 & 5.2 & 10.3 & 7.7 & 9.1 & 3.6 & 10.8 & 7.4 & 9.8 & 6.9 & 8.3 & 4.0 & 12.9 & 6.7 & 8.8 & 13.5 \\
\hline 5 & 417 & $\sigma^{7}$ & $\mathrm{~N}$ & $\mathrm{~N}$ & 18.4 & 19.6 & 9.2 & 9.9 & 6.8 & 17.6 & 12.5 & 9.5 & 11.4 & 14.4 & $\mathrm{~N}$ & $\mathrm{~N}$ & 28.4 & 23.8 & 14.5 & 15.8 & 4.3 \\
\hline 6 & 191 & 우 & 1.3 & 1.0 & 2.8 & 5.7 & 4.0 & 4.0 & 7.0 & 3.9 & 5.3 & 4.6 & 2.4 & 6.0 & 2.6 & 4.4 & 6.5 & 6.8 & 4.0 & 4.6 & 7.0 \\
\hline 7 & 216 & 우 & 5.4 & 3.5 & 3.8 & 8.2 & 8.2 & 1.2 & 10.0 & 0.6 & 0.7 & 6.7 & 7.0 & 4.3 & 4.4 & 3.6 & 7.4 & 0.0 & 5.9 & 3.5 & 25.5 \\
\hline 8 & 223 & 우 & 0.7 & 2.3 & 7.0 & 4.8 & 5.4 & 4.0 & 5.5 & 5.0 & 1.8 & 4.9 & 7.4 & 4.6 & 4.1 & 4.3 & 6.3 & 8.0 & 4.8 & 4.7 & 1.1 \\
\hline 9 & 228 & 우 & 3.0 & 2.7 & 5.3 & 4.8 & 4.1 & 6.0 & 3.0 & 4.8 & 6.6 & 2.7 & 8.2 & 5.2 & 6.1 & 3.0 & 6.4 & 5.4 & 5.3 & 4.3 & 10.4 \\
\hline 10 & 249 & 우 & 0.0 & 2.4 & 5.9 & 7.7 & 2.2 & 8.2 & 2.9 & 8.0 & 6.7 & 2.8 & 8.4 & 6.7 & 8.0 & 1.7 & 7.4 & 6.0 & 5.2 & 5.4 & 1.9 \\
\hline
\end{tabular}

$\mathrm{N}$, Not observed (Next day'intake is for 2 days).

Average

17.9

The other explanations are same as Table 1. 
Table 3. Daily intake and A-index of red-colored and uncolored seeds in the choice test in Rattus rattus.

\begin{tabular}{|c|c|c|c|c|c|c|c|c|c|c|c|c|c|c|c|c|c|c|c|c|c|}
\hline \multirow{3}{*}{$\begin{array}{l}\text { No. } \\
\text { of } \\
\text { rats }\end{array}$} & \multirow{3}{*}{$\begin{array}{l}\text { Body } \\
\text { weight } \\
\text { (g) }\end{array}$} & \multirow{3}{*}{ Sex } & \multicolumn{14}{|c|}{ Daily intake (g) } & \multirow{2}{*}{\multicolumn{4}{|c|}{ Average intake (g/day) }} & \multirow{3}{*}{ A-index } \\
\hline & & & \multicolumn{2}{|c|}{1} & \multicolumn{2}{|c|}{2} & \multicolumn{2}{|c|}{3} & \multicolumn{2}{|c|}{4} & \multicolumn{2}{|c|}{5} & \multicolumn{2}{|c|}{6} & \multicolumn{2}{|c|}{7} & & & & & \\
\hline & & & $\mathrm{R}$ & $\mathrm{U}$ & $\mathrm{R}$ & $\mathrm{U}$ & $\mathrm{R}$ & $\mathrm{U}$ & $\mathrm{R}$ & $\mathrm{U}$ & $\mathrm{R}$ & $\mathrm{U}$ & $\mathrm{R}$ & $\mathrm{U}$ & $\mathrm{R}$ & $\mathrm{U}$ & $\mathrm{R}$ & $\mathrm{U}$ & Le. & Ri. & \\
\hline 1 & 85 & $0^{7}$ & $\mathrm{~N}$ & $\mathrm{~N}$ & $\underline{0.2}$ & 0.7 & 0.4 & $\underline{0.0}$ & $\underline{0.1}$ & 0.0 & 0.0 & $\underline{0.0}$ & $\underline{0.3}$ & 0.2 & 0.0 & $\underline{0.1}$ & 0.2 & 0.2 & 0.1 & 0.2 & - \\
\hline 2 & 85 & $0^{7}$ & $\mathrm{~N}$ & $\mathrm{~N}$ & $\overline{2.6}$ & $\underline{6.5}$ & 3.1 & $\overline{1.4}$ & $\overline{1.3}$ & $\underline{2.8}$ & $\underline{3.2}$ & $\overline{1.0}$ & $\overline{2.2}$ & $\underline{2.7}$ & $\underline{2.2}$ & $\overline{1.4}$ & 2.4 & 2.6 & 3.4 & 1.7 & -4.0 \\
\hline 3 & 87 & $0^{7}$ & $\mathrm{~N}$ & $\mathrm{~N}$ & 1.4 & $\overline{6.1}$ & $\overline{4.0}$ & 0.0 & 0.0 & $\overline{4.8}$ & $\overline{3.6}$ & 0.6 & 0.5 & $\overline{4.8}$ & $\overline{2.2}$ & 1.2 & 2.0 & 2.9 & 4.3 & 0.6 & -18.4 \\
\hline 4 & 90 & $0^{7}$ & $\mathrm{~N}$ & $\mathrm{~N}$ & 1.6 & $\overline{9.7}$ & $\overline{2.8}$ & 3.8 & 4.2 & $\overline{1.2}$ & $\overline{1.6}$ & 2.4 & 2.4 & $\overline{2.0}$ & $\overline{1.7}$ & 2.0 & 2.4 & 3.5 & 2.7 & 3.2 & -18.6 \\
\hline 5 & 101 & $0^{x}$ & $\mathrm{~N}$ & $\mathrm{~N}$ & 1.6 & 2.8 & 2.8 & $\underline{1.0}$ & $\overline{2.2}$ & 0.9 & 1.2 & $\overline{2.0}$ & 1.0 & 1.7 & 1.4 & $\underline{1.2}$ & 1.7 & 1.6 & 1.5 & 1.8 & 3.0 \\
\hline 6 & 65 & 우 & $\mathrm{N}$ & $\mathrm{N}$ & $\overline{2.7}$ & 7.4 & 3.2 & $\overline{1.7}$ & $\overline{0.6}$ & 3.8 & 3.9 & $\overline{0.5}$ & $\underline{0.6}$ & 3.5 & 3.7 & $\overline{0.3}$ & 2.5 & 2.9 & 1.1 & 4.3 & -7.4 \\
\hline 7 & 65 & 우 & $\mathrm{N}$ & $\mathrm{N}$ & $\overline{2.3}$ & 2.6 & 1.2 & $\overline{0.1}$ & $\overline{0.2}$ & $\underline{0.6}$ & $\underline{2.2}$ & $\overline{0.9}$ & $\overline{0.3}$ & $\underline{4.0}$ & $\underline{2.8}$ & $\overline{0.7}$ & 1.5 & 1.5 & 2.2 & 0.8 & 0.0 \\
\hline 8 & 70 & 우 & $\mathrm{N}$ & $\mathrm{N}$ & 3.5 & $\overline{2.7}$ & $\overline{0.5}$ & 3.9 & 1.3 & $\overline{0.0}$ & $\overline{0.0}$ & 0.0 & $\mathrm{E}$ & $\mathrm{E}$ & $\mathrm{E}$ & $\mathrm{E}$ & 1.3 & 1.7 & 0.8 & 2.2 & -13.3 \\
\hline 9 & 89 & 우 & $\mathrm{N}$ & $\mathrm{N}$ & $\underline{6.4}$ & $\overline{3.7}$ & $\overline{3.8}$ & $\underline{0.1}$ & $\underline{0.8}$ & $\overline{3.2}$ & $\overline{4.0}$ & $\underline{1.0}$ & $\underline{4.3}$ & 1.3 & 0.9 & 3.8 & 3.4 & 2.2 & 2.7 & 2.8 & 21.4 \\
\hline 10 & 104 & 우 & $\mathrm{N}$ & $\mathrm{N}$ & $\overline{2.9}$ & $\underline{5.2}$ & 2.5 & $\overline{2.5}$ & $\overline{3.1}$ & 1.4 & $\underline{0.7}$ & $\overline{3.4}$ & $\overline{3.0}$ & $\underline{1.3}$ & $\underline{1.3}$ & $\overline{3.4}$ & 2.3 & 2.9 & 2.1 & 3.1 & -11.5 \\
\hline
\end{tabular}

R, Red bait; U, Uncolored bait.

Under line, Left side (Le.); No mark, Right side (Ri.).

A-index of average intake $=\{(\mathrm{R}-\mathrm{U}) /(\mathrm{R}+\mathrm{U})\} \times 100$.

- , The data was not adopted.

N, Not observed.

E, Escaped. 
Table 4. Daily intake and A-index of red-colored and uncolored feed in the choice test in Rattus norvegicus.

\begin{tabular}{|c|c|c|c|c|c|c|c|c|c|c|c|c|c|c|c|c|c|c|c|c|c|c|c|}
\hline \multirow{3}{*}{$\begin{array}{l}\text { No. } \\
\text { of } \\
\text { rats }\end{array}$} & \multirow{3}{*}{$\begin{array}{l}\text { Body } \\
\text { weight } \\
\text { (g) }\end{array}$} & \multirow{3}{*}{ Sex } & \multicolumn{16}{|c|}{ Daily intake (g) } & \multirow{2}{*}{\multicolumn{4}{|c|}{ Average intake (g/day) }} & \multirow{3}{*}{ A-index } \\
\hline & & & \multicolumn{2}{|c|}{1} & \multicolumn{2}{|c|}{2} & \multicolumn{2}{|c|}{3} & \multicolumn{2}{|c|}{4} & \multicolumn{2}{|c|}{5} & \multicolumn{2}{|c|}{6} & \multicolumn{2}{|c|}{7} & \multicolumn{2}{|c|}{8} & & & & & \\
\hline & & & $\mathrm{R}$ & $\mathrm{U}$ & $\mathrm{R}$ & $\mathrm{U}$ & $\mathrm{R}$ & $\mathrm{U}$ & $\mathrm{R}$ & $\mathrm{U}$ & $\mathrm{R}$ & $\mathrm{U}$ & $\mathrm{R}$ & $\mathrm{U}$ & $\mathrm{R}$ & $\mathrm{U}$ & $\mathrm{R}$ & $\mathrm{U}$ & $\mathrm{R}$ & $\mathrm{U}$ & Le. & Ri. & \\
\hline 1 & 307 & $0^{x}$ & 20.0 & 9.2 & 20.7 & 6.8 & 8.7 & 14.5 & 19.6 & 6.0 & 2.8 & 20.1 & $\underline{\mathrm{N}}$ & $\underline{N}$ & 40.3 & $\underline{3.3}$ & $\underline{1.8}$ & 15.2 & 16.3 & 10.7 & 7.1 & 19.9 & 20.7 \\
\hline 2 & 347 & $0^{\pi}$ & $\overline{3.3}$ & 1.6 & 1.7 & $\overline{3.0}$ & $\overline{3.7}$ & 1.8 & 10.1 & $\overline{5.5}$ & $1 \overline{5.3}$ & 6.9 & $\overline{\mathrm{N}}$ & $\overline{\mathrm{N}}$ & 16.7 & $\overline{26.3}$ & 16.9 & 1.1 & 9.7 & 6.6 & 8.0 & 8.3 & 19.0 \\
\hline 3 & 186 & 우 & 2.3 & $\overline{9.6}$ & $1 \overline{4.0}$ & 4.1 & 5.4 & $2 \overline{1.4}$ & $\overline{10.4}$ & 10.1 & 11.7 & $1 \overline{6.9}$ & $\overline{\mathrm{N}}$ & $\overline{\mathrm{N}}$ & $\overline{39.6}$ & 3.4 & $\overline{0.5}$ & 19.9 & 12.0 & 12.2 & 5.4 & 18.8 & -0.8 \\
\hline 4 & 237 & 우 & 1.1 & 1.4 & 1.5 & 1.7 & $\frac{1.5}{1.5}$ & 1.7 & 1.0 & 1.5 & 1.2 & 1.0 & $\overline{\mathrm{N}}$ & $\overline{\mathrm{N}}$ & 1.3 & $\overline{1.4}$ & $\overline{0.1}$ & 0.2 & 1.1 & 1.3 & 1.2 & 1.2 & -8.3 \\
\hline 5 & 256 & 우 & $\underline{1.6}$ & $\overline{2.7}$ & $\overline{4.7}$ & $\underline{2.1}$ & $\underline{12.1}$ & $\overline{5.7}$ & $\overline{19.7}$ & 1.6 & $\underline{16.1}$ & $\overline{4.2}$ & $\underline{\mathrm{N}}$ & $\underline{\bar{N}}$ & $\overline{38.1}$ & 3.4 & $\underline{9.4}$ & $\overline{6.8}$ & 14.5 & 3.8 & 6.6 & 11.7 & 58.5 \\
\hline
\end{tabular}

The explanations are same as Table 3. 
Table 5. Daily intake and A-index of whole rice flour and mixed powdered feed in the choice test in Rattus rattus.

\begin{tabular}{|c|c|c|c|c|c|c|c|c|c|c|c|c|c|c|c|c|c|c|c|c|c|}
\hline \multirow{3}{*}{$\begin{array}{l}\text { No. } \\
\text { of } \\
\text { rats }\end{array}$} & \multirow{3}{*}{$\begin{array}{l}\text { Body } \\
\text { weight } \\
(\mathrm{g})\end{array}$} & \multirow{3}{*}{ Sex } & \multicolumn{14}{|c|}{ Daily intake (g) } & \multirow{2}{*}{\multicolumn{4}{|c|}{ Average intake (g/day) }} & \multirow{3}{*}{ A-index } \\
\hline & & & \multicolumn{2}{|c|}{1} & \multicolumn{2}{|c|}{2} & \multicolumn{2}{|c|}{3} & \multicolumn{2}{|c|}{4} & \multicolumn{2}{|c|}{5} & \multicolumn{2}{|c|}{6} & \multicolumn{2}{|c|}{7} & & & & & \\
\hline & & & W & M & W & M & W & M & W & $\mathrm{M}$ & W & M & W & $\mathrm{M}$ & W & M & W & M & Le. & Ri. & \\
\hline 1 & 85 & $\sigma^{\pi}$ & $\underline{0.6}$ & 0.7 & 2.0 & 4.9 & 2.0 & 5.5 & 0.4 & 8.5 & 1.8 & 6.1 & 0.1 & $\underline{7.7}$ & 1.2 & 5.9 & 1.2 & 5.6 & 3.8 & 3.0 & -64.7 \\
\hline 2 & 85 & $\sigma^{\pi}$ & $\overline{2.6}$ & $\underline{0.4}$ & 6.8 & $\overline{1.4}$ & $\overline{6.1}$ & 1.9 & 5.4 & $\overline{1.9}$ & $\overline{6.9}$ & 1.4 & 4.8 & $\overline{1.7}$ & $\overline{4.7}$ & 3.6 & 5.3 & 1.8 & 3.5 & 3.6 & 49.3 \\
\hline 3 & 87 & $\sigma^{\pi}$ & 0.3 & $\overline{2.4}$ & $\overline{1.9}$ & 4.6 & 0.9 & $\overline{5.6}$ & $\overline{2.6}$ & 4.2 & 1.8 & $\overline{5.2}$ & $\overline{5.0}$ & 0.8 & 3.5 & $\overline{3.6}$ & 2.3 & 3.8 & 3.8 & 2.3 & -24.6 \\
\hline 4 & 90 & $0^{\pi}$ & 0.0 & 0.0 & 0.0 & 0.0 & 0.0 & $\overline{0.0}$ & 0.0 & 0.0 & 0.0 & $\overline{5.7}$ & $\overline{0.0}$ & 7.0 & 0.0 & $\overline{9.8}$ & 0.0 & 3.2 & 1.0 & 2.2 & -100.0 \\
\hline 5 & 101 & $\sigma^{\pi}$ & $\overline{0.6}$ & 3.8 & 0.8 & $\underline{5.2}$ & $\overline{0.0}$ & 3.7 & 0.2 & $\overline{5.6}$ & $\overline{0.2}$ & 4.1 & 0.0 & $\overline{5.0}$ & $\overline{0.0}$ & 4.7 & 0.3 & 4.6 & 2.4 & 2.5 & -87.8 \\
\hline 6 & 65 & 우 & $\overline{0.1}$ & 5.0 & 0.5 & $\overline{7.3}$ & $\overline{0.2}$ & 7.7 & 0.2 & $\overline{8.0}$ & $\overline{0.0}$ & 8.7 & 0.0 & $\overline{7.3}$ & $\overline{0.0}$ & 7.4 & 0.1 & 7.3 & 3.3 & 4.2 & -97.3 \\
\hline 7 & 65 & 우 & $\overline{0.2}$ & 2.5 & 4.6 & $\overline{0.0}$ & $\overline{5.3}$ & 0.1 & 5.8 & $\overline{0.0}$ & $\overline{6.5}$ & 0.1 & 6.4 & $\overline{0.0}$ & $\overline{5.2}$ & 0.1 & 4.9 & 0.4 & 2.8 & 2.5 & 84.9 \\
\hline 8 & 70 & 우 & 1.1 & $\overline{3.1}$ & $\overline{2.8}$ & 3.9 & 3.0 & $\overline{3.4}$ & $\overline{3.1}$ & 3.6 & 2.7 & $\overline{4.8}$ & $\overline{2.3}$ & 4.9 & 1.5 & $\overline{5.7}$ & 2.4 & 4.2 & 3.6 & 3.0 & -27.3 \\
\hline 9 & 89 & 우 & 2.3 & $\overline{3.6}$ & $\overline{3.8}$ & 5.3 & 3.1 & $\overline{5.9}$ & $\overline{6.2}$ & 3.6 & 6.2 & $\overline{3.2}$ & $\overline{5.2}$ & 3.7 & 6.2 & $\overline{1.8}$ & 4.7 & 3.9 & 4.3 & 4.2 & 9.3 \\
\hline 10 & 104 & 우 & $\overline{0.2}$ & $\underline{1.4}$ & $\underline{4.2}$ & $\overline{1.4}$ & $\overline{4.8}$ & 3.4 & $\underline{7.8}$ & $\overline{0.6}$ & $\overline{5.0}$ & $\underline{4.1}$ & $\underline{6.3}$ & $\overline{1.6}$ & $\overline{5.5}$ & $\underline{2.4}$ & 4.8 & 2.1 & 4.2 & 2.7 & 39.1 \\
\hline
\end{tabular}

W, Whole rice flour; $\mathrm{M}$, Mixed powdered feed.

A-index of average intake $=\{(\mathrm{W}-\mathrm{M}) /(\mathrm{W}+\mathrm{M})\} \times 100$.

Under line is same as Table 3. 
Table 6. Figures of A-index.

\begin{tabular}{crrrrr}
\hline \hline Table & S. size & Ave. & SD & SE & $\begin{array}{c}\text { 95\% confidence } \\
\text { limit }\end{array}$ \\
\hline 1 & 10 & 22.1 & 15.0 & 4.7 & $11.4 \sim 32.8$ \\
2 & 10 & 17.9 & 16.4 & 5.2 & $6.1 \sim 29.7$ \\
3 & 9 & -5.4 & 12.6 & 4.2 & $-15.1 \sim 4.3$ \\
4 & 5 & 17.8 & 25.9 & 11.6 & $-14.4 \sim 50.0$ \\
5 & 10 & -21.9 & 66.0 & 20.9 & $-69.1 \sim 25.3$ \\
\hline
\end{tabular}

S. size, Sample size

Emer. Tyuji Kusano, University of Tsukuba, for useful suggestions regarding this study.

\section{REFERENCES}

Ikeda, Y., Tabaru, Y., Yuyama, Y. and Naganuma, K. 1976. Studies on the food habits of rats. III. Feeding preference of wild Norway rats in various habitats. Botyu-Kagaku, 41: 75-77.

Ito, Y. 1998. A study on acceptability of roof rats, Rattus rattus to rodenticidal baits Part 2. Med. Entomol. Zool., 50th Ann. Meet.: 52 (in Japanese).

Ito, Y. and Motoki, M. 1982. Feeding preference of roof rats, Rattus rattus to several food materials. Annu. Rep. JESC., 9: 73-75 (in Japanese with English summary).
Jain, A. P. and Pawde, V. A. 1981. Responses of a free-living population of Rattus rattus (Linn.) towards poisoned baits and new food. Indian J. Exp. Biol., 19: 678-679.

Naganuma, K., Sakurai, T. and Ikeda, Y. 1973. Studies on the food habits of rats. I. Feeding preferences of the Norway rats on some unexperienced diets. Jpn. J. Sanit. Zool., 24: 23-26 (in Japanese with English summary).

Nahas, K., Lorgue, G. and Mazallon, M. 1989. Difethialone (LM-2219): a new anticoagulant rodenticide for use against warfarin-resistant and-susceptible strains of Rattus norvegicus and Mus musculus. Ann. Rech. Vet., 20: 159-164.

Ogushi, K. and Tokumitsu, I. 1970. Studies on rodenticides (I) Feeding preference of Norway rats on dry baits with various materials and forms. Jpn. J. Sanit. Zool., 21: 107-111 (in Japanese with English summary).

Prakash, I., Advani, R., Soni, B. K. and Rana, B. D. 1980. Evaluation of the bait base for the control of common house rat, Rattus rattus rufescens (Gray). Z. Angew. Zool., 67(2): 211-223.

Shafi, M. M., Ahmed, S. M., Pervez, A. P. and Ahmad, S. 1992. Enhancement of poison bait acceptance through taste additives in Rattus norvegicus. J. Stored Prod. Res., 28(4): 239-243. 